\title{
Distinction of subtype-specific antibodies against European porcine influenza viruses by indirect ELISA based on recombinant hemagglutinin protein fragment-1
}

Na Zhao ${ }^{1}$, Elke Lange ${ }^{2}$, Sybille Kubald ${ }^{3}$, Christian Grund ${ }^{1}$, Martin Beer ${ }^{1}$ and Timm C Harder ${ }^{1 *}$

\begin{abstract}
Background: Serological investigations of swine influenza virus infections and epidemiological conclusions thereof are challenging due to the complex and regionally variable pattern of co-circulating viral subtypes and lineages and varying vaccination regimes. Detection of subtype-specific antibodies currently depends on hemagglutination inhibition (HI) assays which are difficult to standardize and unsuitable for large scale investigations.

Methods: The nucleocapsid protein (NP) and HA1 fragments of the hemagglutinin protein (HA) of five different lineages (H1N1av, H1N1pdm, H1pdmN2, H1N2, H3N2) of swine influenza viruses were bacterially expressed and used as diagnostic antigens in indirect ELISA.

Results: Proteins were co-translationally mono-biotinylated and refolded in vitro into an antigenically authentic conformation. Western blotting and indirect ELISA revealed highly subtype-specific antigenic characteristics of the recombinant $\mathrm{HA} 1$ proteins although some cross reactivity especially among antigens of the $\mathrm{H} 1$ subtype were evident. Discrimination of antibodies directed against four swine influenza virus subtypes co-circulating in Germany was feasible using the indirect ELISA format.

Conclusions: Bacterially expressed recombinant NP and HA1 swine influenza virus proteins served as antigens in indirect ELISAs and provided an alternative to commercial blocking NP ELISA and HI assays concerning generic (NP-specific) and HA subtype-specific sero-diagnostics, respectively, on a herd basis.
\end{abstract}

Keywords: Pandemic influenza, Swine, Mixing vessel, Subtype specificity, Sero-diagnostics

\section{Background}

Swine influenza is an economically important disease of pigs caused by infections with influenza A viruses (IAV). Economic losses are caused by retarded growth of fattening pigs due to influenza-induced or -aggravated respiratory disease. In addition, febrile influenza virus infections in sows may cause fertility problems [1]. Influenza viruses are an important factor in the polymicrobial respiratory syndrome of swine [2]. In contrast to human influenza, infections in swine do not appear to be seasonal, and virus circulation, especially in larger herds, is observed

\footnotetext{
* Correspondence: timm.harder@fli.bund.de

${ }^{1}$ Institute of Diagnostic Virology, Friedrich-Loeffler-Institut, Suedufer 10, Greifswald 17493, Germany

Full list of author information is available at the end of the article
}

year-round [3]. Control of swine influenza is difficult and requires strict zoosanitary measures and herd vaccination programs. Licensed inactivated whole virus vaccines are available but high and continuous vaccine coverage within herds is required [4].

In addition to being affected by disease, pigs are considered to be an integral part of the wider epidemiology of influenza, bridging the avian influenza world to mammalian influenza. The porcine respiratory epithelium is lined by cells which express the two sialic acid glycan receptor structures to which avian- or mammalian-adapted IAV bind [5]. As such, pigs can be infected by IAV of human and of avian origin and this provides opportunities for reassortment between these viruses [6,7]. Following historic transspecies transmission events of IAV

\section{Biomed Central}


from human or avian sources to pigs some of these viruses have established stable circulating lineages in swine populations worldwide. These porcine lineages continue to reassort amongst each other and with other IAV of more recent human or avian origin. In Europe, this scenario has lead to current presence of at least four distinct lineages of swine influenza viruses in pig populations $[8,9]$.

Since the late 1970s IAV of subtype H1N1 of purely avian origin (H1N1av) dominate the influenza epidemiology in swine in many European countries including Germany. This lineage, referred to as H1N1av, has fully adapted to swine and can be distinguished genetically and antigenically from current avian-adapted H1N1 viruses. Viruses of this lineage have sporadically been detected also in humans and turkeys in Europe due to single transmission events from infected pigs [10,11]. A second porcine lineage consists of viruses of subtype H3N2. The progenitor of the currently circulating porcine H3N2 strains originated from human-adapted H3N2 viruses which had caused the Hong Kong flu pandemic in 1968 [8]. In the early 1980 s the descendants of this virus reassorted with H1N1av and, apart from the hemagglutinin $\mathrm{H} 3$ and the neuraminidase N2 segments, all further six genome segments were replaced with those of H1N1av [8]. In the early 1990s, a new porcine triple reassortant virus, H1N2, arose from reassortment events between human seasonal $\mathrm{H} 1 \mathrm{~N} 1$ and $\mathrm{H} 3 \mathrm{~N} 2$ and porcine H1N1av viruses. This porcine $\mathrm{H} 1 \mathrm{~N} 2$ virus carried hemagglutinin (HA) and neuraminidase (NA) of human origin and the cassette of six further segments of H1N1av [8]. These three lineages continue to co-circulate at varying prevalences in different European countries.

In 2009, a new human pandemic H1N1 strain (H1N1pdm) emerged. This virus carried reassorted gene segments from several American and Eurasian swine influenza lineages and was rapidly introduced from the human population to pigs [8]. Pigs proved to be highly susceptible to this virus and stable transmission chains were easily maintained [12]. To date H1N1pdm appears to circulate independently from the human population in swine in several countries worldwide. Recently we and others found evidence for the emergence of reassortants between H1N1pdm and authentic porcine influenza virus lineages in Germany. In particular, a reassortant lineage of subtype H1pdmN2 which carried seven segments of the H1N1pdm virus and a neuraminidase of subtype 2 that was derived from different porcine or human $\mathrm{HxN} 2$ lineages, circulated stably $[9,13]$.

Measures aiming at control of swine influenza must be based on subtype-specific virological and serological diagnosis. Real-time RT-PCR (RT-qPCR) has provided ample applications for rapid and sensitive molecular virological diagnosis [14]. Serology in swine influenza has been found useful for retrospective epidemiological investigations e.g., [15], estimation of disease incidence e.g., [16], and control of vaccination success e.g., [17]. Several commercial ELISAs for detection of generic IAV nucleocapsid protein (NP)-specific antibodies in pigs are available and recommended for use e.g., [18]. With regard to detection of antibodies at the subtype-specific level the hemagglutination inhibition assay (HI) is still held gold standard despite several draw-backs of this method including timeand labour-consuming performance and dependence on labile and difficult-to-standardize components (viral antigen, erythrocytes). Furthermore, due to interference of antibodies against the viral neuraminidase component interpretation of HI-results are particularly difficult $[19,20]$. Antibodies to the IAV hemagglutinin protein (HA) become detectable by $\mathrm{HI}$ assay from the second week post infection on and $\mathrm{HI}$ titers correlate with protection from clinically overt disease $[21,22]$.

Despite mentioned problems of $\mathrm{HI}$ assays very few swine influenza ELISA applications aiming at subtype differentiation at the antibody level have been reported and the current commercially available assays for subtypes $\mathrm{H} 1$ and $\mathrm{H} 3$ have not superseded $\mathrm{HI}$ assays, at least in Europe [23]. The reported lack of sensitivity of these assays may be related to the American origin of the IAV isolates used which are antigenically distinct from those circulating in Europe. Low specificity of these assays may be caused by use of whole virion preparations which contain group specific antigens such as the nucleocapsid protein.

Here we show that recombinant HA1 antigen of European swine influenza viruses which was bacterially expressed and refolded in vitro can be used in indirect ELISAs for detection and differentiation of subtype-specific antibodies in porcine sera.

\section{Results}

\section{Bacterial expression of antigenic influenza HA1 protein}

The HA1 protein fragments of seven recent swine influenza virus isolates (Table 1) were bacterially expressed (pET19b expression vectors) and co-translationally monobiotinylated by overexpressed bacterial BirA biotin ligase (pBIRAcm vector). In addition, the full-length nucleocapsid protein of one of the seven isolates was expressed similarly. The recombinant proteins sequestered into bacterial inclusion bodies. Purified bacterial inclusion body proteins were subjected to SDS-PAGE under reducing conditions for detection by Western blot analysis (Figure 1). Using an anti-biotin monoclonal antibody, recombinant proteins of expected molecular weights (HA-1 $38+/-3 \mathrm{kD}$; $\mathrm{NP}$ ca, $56 \mathrm{kD}$ ) are depicted in Figure 1A. No further protein bands were identified and no biotinylated proteins were detected in a control which consisted of a clarified lysate of Rosettagami E. coli cells which had 
Table 1 Origin and properties of porcine influenza viruses used in this study for generation of recombinant proteins

\begin{tabular}{lcccc}
\hline Identification & Subtype & HA $^{\text {a Lineage }}$ & Sequence HA & Sequence NP \\
\hline A/Germany/R26/2011 & H1N1pdm & Pandemic 2009 & EPI356430 & n.d. \\
A/swine/Germany/R1738/2010 & H1N1 & Eurasian avian-like & EPI411955 & EPI426141 \\
A/swine/Germany/R1931/2011 & H1N2 & Eurasian avian-like reassortant & EPI412039 & n.d. \\
A/swine/Germany/R1207/2010 & H1N2 & Eurasian human-like reassortant & EPI411941 & n.d. \\
A/swine/Germany/R2035/2011 & H1pdmN2 & Pandemic 2009 reassortant & EPI356453 & n.d. \\
A/swine/Germany/R96/2011 & H3N2 & Eurasian human-like reassortant & EPI411978 & n.d. \\
A/swine/Germany/R76/2011 & H3N2 & Eurasian human-like reassortant & EPI411965 & n.d. \\
\hline
\end{tabular}

${ }^{\mathrm{a}} \mathrm{HA}$ - hemagglutinin.

${ }^{b} \mathrm{NP}$ - nucleocapsid.

c Sequence accession number in EpiFlu database.

n.d. - not determined.

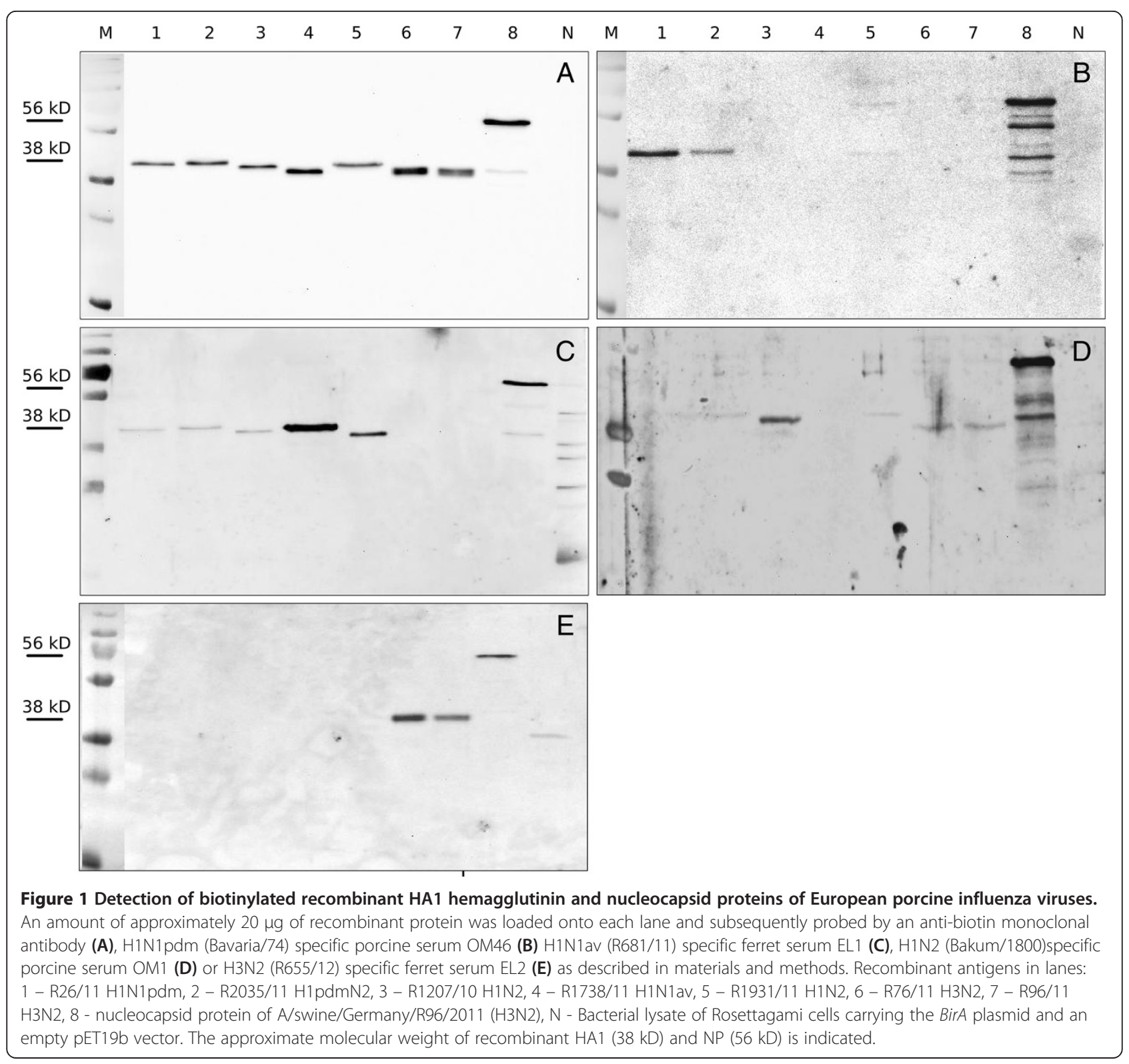


been co-transformed by an empty pET19b expression vector and pBIRAcm. The NP protein showed liability to proteolytical degradation as shown by a few and weak bands of lower molecular weight (Figure 1A, lane 8). Thus, the chosen bacterial co-expression system specifically produced biotinylated recombinant HA1 and NP proteins which could be successfully purified from inclusion bodies.

The antigens reacted also with sera from IAV infected pigs and ferrets (Figure 1B-E; Table 2). The NP-antigen, although derived from a porcine $\mathrm{H} 3 \mathrm{~N} 2$ virus, was recognized by sera raised against four porcine IAV lineages (H1N1pdm, H1N1av, H1N2, and the homologous H3N2) as shown each in lanes 8 of Figure 1, panels B - D. A porcine serum raised against $\mathrm{H} 1 \mathrm{~N} 1 \mathrm{pdm}$ was specific for the HA1 proteins of $\mathrm{H} 1 \mathrm{~N} 1 \mathrm{pdm}$ and the reassortant H1pdmN2 (Figure 1B, lane 1 and 2). Serum from a ferret experimentally infected by an H1N1av isolate strongly reacted with homologous H1av HA1 proteins (Figure 1C, lane 4) but cross-reacted weakly also with other H1 HA1 recombinant proteins. An H1N2-specific porcine serum (Figure 1D) similarly showed strong specific staining with the homologous H1N2 HA1 (lane 3) and produced weaker signals with other recombinant HA1 antigens (e.g., lanes 2, 5). A ferret anti-H3 serum proved to be subtype-specific (Figure 1E, lanes 6 and 7).

\section{Determination of cut-off values for indirect ELISAs using recombinant NP and HA1 proteins}

Using the biotin residue of the recombinant NP and HA1 proteins, streptavidin ELISA plates were coated with $0,5 \mu \mathrm{g}$ of recombinant protein per well and then blocked. Sera to be tested were prediluted 1:200 and species- specific anti IgG antibodies conjugated with horseraddish peroxidase were used to detect bound antibodies in this indirect ELISA format. Seventy five sera from a total of 50 pigs were examined to determine cut-off values. These pigs originated from the influenza-negative minipig breeding cluster at FLI $(\mathrm{n}=10)$ and from a swine farm which tested continuously seronegative against IAV. None of these sera tested positive in a commercial NP-specific blocking ELISA (ID.Vet, France). Mean extinctions were measured and standard deviations were calculated for the indirect ELISAs using recombinant NP and HA1 (Table 3). A porcine serum derived from a pig after multiple vaccination with four subtypes of European swine IAV possessed high $\mathrm{HI}$ titres against H1av, H1N2, H1pdm and H3N2 (Table 2) and was chosen as standard positive control. Likewise a negative porcine field serum with OD values close to the upper cut-off value of the set of negative sera was chosen as standard negative control. Based on these control sera (Table 3) S/P ratios were calculated for the set of 75 negative sera and the S/P mean plus 2 SD (recombinant HA1) or $3 \mathrm{SD}$ (recombinant NP) were chosen as cut-offs in the examination of porcine field sera by indirect ELISAs. The exact cut-off values for each assay are presented in Table 3.

\section{Specificity of HA1-specific indirect ELISAs}

Subsequently recombinant HA1-antigens were used to test sera obtained from experimentally infected pigs at day 21 after inoculation (Figure 2). HI assays confirmed a high specificity for these sera but also revealed minor cross reactivities especially between sera raised against subtype $\mathrm{H} 1$ viruses (Table 2). This was reflected in indirect ELISA in which the highest $\mathrm{S} / \mathrm{P}$ ratios (set to 1.0)

Table 2 HI titres of porcine and ferret post infection sera used in Western blotting and indirect ELISA (homologous serum-antigen pairs depicted in bold)

\begin{tabular}{|c|c|c|c|c|c|c|c|}
\hline \multirow[t]{2}{*}{ Serum raised against } & \multirow[t]{2}{*}{ Subtype } & \multirow[t]{2}{*}{ Host } & \multicolumn{5}{|c|}{ Hemagglutination inhibiting titre } \\
\hline & & & $\mathrm{H}_{1 N 1 a v}{ }^{a}$ & $\mathrm{H} 1 \mathrm{~N} 2^{\mathrm{b}}$ & H1N1pdm c & $\mathrm{H} 1 \mathrm{pdmN} 2^{\mathrm{d}}$ & $\mathrm{H} 3 \mathrm{~N} 2^{\mathrm{e}}$ \\
\hline A/swine/Belzig/2/2001 & H1N1av & Swine (OM8) & $4^{*}$ & 1 & 2 & 1 & 1 \\
\hline A/swine/Germany/ R681/2011 & H1N1av & Ferret (EL1) & 6 & 1 & 6 & 2 & 2 \\
\hline A/swine/Bakum/1832/ 2000 & $\mathrm{H} 1 \mathrm{~N} 2$ & Swine (OM1) & 1 & 5 & 1 & 1 & 1 \\
\hline A/swine/Bayern/74/ 2009 & H1N1pdm & Swine $(\mathrm{OM} 46)^{f}$ & 4 & 1 & 6 & 5 & 1 \\
\hline A/swine/Germany/ R2035/2011 & H1pdmN2 & Swine (OM15/4) & 1 & 3 & 5 & 6 & 1 \\
\hline A/swine/Belzig/54/ 2001 & $\mathrm{H} 3 \mathrm{~N} 2$ & Swine (R4) & 1 & 1 & 1 & 1 & 6 \\
\hline A/swine/Germany/ R655/2012 & $\mathrm{H} 3 \mathrm{~N} 2$ & Ferret (EL1) & 1 & 1 & 2 & 2 & 9 \\
\hline Unknown, field serum & Pan & swine & 6 & 6 & 5 & 5 & 6 \\
\hline Unknown, field serum & None & swine & 1 & 1 & 1 & 1 & 1 \\
\hline
\end{tabular}

Viruses that had been used for production of recombinant HA1 proteins were also used in HI assays:

a - A/swine/Germany/R1738/2010.

b - A/swine/Germany/R1207/2010.

c - A/Germany/R26/2011.

d - A/swine/Germany/R2035/2011.

e - A/swine/Germany/R96/2011.

${ }^{*}$ - $\log _{2}$ titre (starting dilution 1:10). 
Table 3 Determination of cut-offs of indirect ELISAs based on recombinant NP and HA1 proteins

\begin{tabular}{|c|c|c|c|c|c|c|c|}
\hline \multirow[t]{2}{*}{ Serum samples } & \multirow[t]{2}{*}{ Values } & \multicolumn{6}{|c|}{ Recombinant antigens } \\
\hline & & NP & H1N1av & $\mathrm{H} 1 \mathrm{~N} 2$ & N1N1pdm & H1pdmN2 & $\mathrm{H} 3 \mathrm{~N} 2$ \\
\hline Negative sera & Mean & 0,11 & 0,03 & 0,06 & 0,03 & 0,04 & 0,14 \\
\hline \multirow[t]{2}{*}{$N=75$} & SD & 0,08 & 0,03 & 0,10 & 0,08 & 0,07 & 0,14 \\
\hline & $X+2 S D$ & $0,35^{*}$ & 0,08 & 0,25 & 0,19 & 0,18 & 0,41 \\
\hline \multirow[t]{2}{*}{ Positive standard } & Mean & 0,42 & 094 & 0,61 & 0,92 & 0,54 & 0,58 \\
\hline & SD & 0,02 & 0,01 & 0,01 & 0,01 & 0,02 & 0,02 \\
\hline \multirow[t]{2}{*}{ Negative standard } & Mean & 0,12 & 0,06 & 0,15 & 0,18 & 0,16 & 0,15 \\
\hline & SD & 0,02 & 0,01 & 0,00 & 0,01 & 0,03 & 0,01 \\
\hline
\end{tabular}

Viruses used for production of recombinant proteins:

NP, H1N1av - A/swine/Germany/R1738/2010.

H1N2 - A/swine/Germany/R1207/2010.

H1N1pdm - A/Germany/R26/2011.

H1pdmN2 - A/swine/Germany/R2035/2011.

H3N2 - A/swine/Germany/R96/2011.

* - For NP a cut-off of 3 SD was used.

were consistently obtained with the homologous serumrecombinant HA1 protein pair. However, cross reactions were also evident by indirect ELISA, particularly among proteins of the $\mathrm{H} 1$ lineages. In general, cross reactions resulted in S/P ratios well below 0.5 although a serum raised against pandemic $\mathrm{H} 1$ could not distinguish pandemic $\mathrm{H} 1$ from reassorted $\mathrm{H} 1 \mathrm{pdmN} 2$ and vice versa.
In order to test whether the observed serum cross reactivities were due to low-affinity antibody species, avidity assays were performed. Indirect ELISA assays were extended by an urea washing step for 10 minutes at room temperature before second antibody conjugate was added. As shown in Table 4, urea treatment diminished antibody reactivity against heterologous recombinant HA1

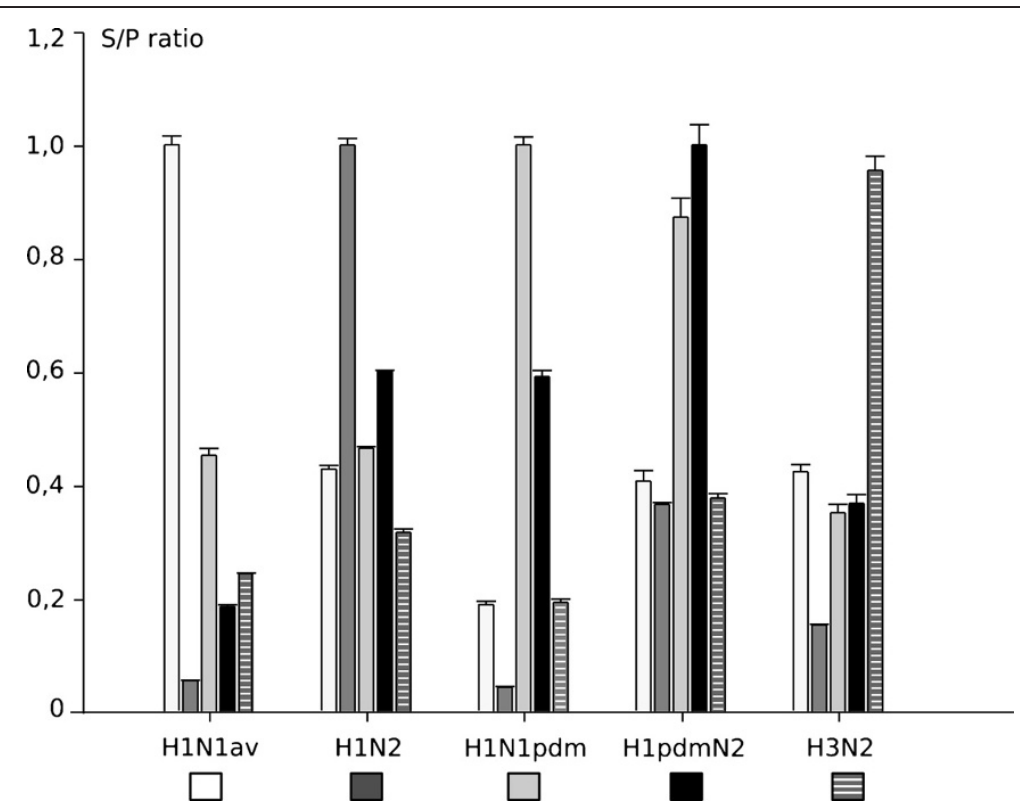

Figure 2 Specificity of an indirect ELISA based on recombinant hemagglutinin fragment HA1 of porcine influenza A viruses. Sera were obtained at day 21 of infection experiments in swine using the following viruses for inoculation: H1N1av - Porcine serum OM8 against A/swine/ Belzig/2/2001, H1N2 - Porcine serum OM1 against A/swine/Bakum/1832/2000, H1N1pdm - Porcine serum OM46 against A/Bayern/74/2009, H1pdmN2 - Porcine serum OM15/4 against A/swine/Germany/R2035/2011, H3N2 - Porcine serum R4 against A/swine/Belzig/54/2001. The recombinant HA1 antigens were produced from the following viruses: H1N1av - A/swine/Germany/R1738/2010; H1N2 - A/swine/Germany/ R1207/2010; H1N1pdm - A/Germany/R26/2011; H1pdmN2 - A/swine/Germany/R2035/2011; H3N2 - A/swine/Germany/96/2011. 
Table 4 Serum avidity in indirect HA1 ELISA

\begin{tabular}{|c|c|c|c|c|c|c|c|c|c|}
\hline \multirow[t]{3}{*}{ Recombinant $\mathrm{HA}$} & \multicolumn{9}{|c|}{ Serum specificity } \\
\hline & \multicolumn{3}{|c|}{ H1N1 pdm-A/Bayern/74/2009 ${ }^{a}$} & \multicolumn{3}{|c|}{ H1N1av -A/swine/Belzig/2/2001 } & \multicolumn{3}{|c|}{ H3N2 - A/swine Belzig/54/2001 } \\
\hline & No urea & Urea & AVI (\%) & No urea & Urea & AVI (\%) & No urea & Urea & AVI (\%) \\
\hline A/Germany/R26/2011 (H1N1pdm) & $2,332^{\mathrm{b}}$ & 1,273 & 55 & 1,011 & 0,163 & 16 & 0,286 & 0,108 & 38 \\
\hline A/Swine/Germany/R2035/2011 (H1 pdmN2) & 1,359 & 0,266 & 20 & 0,292 & 0,109 & 37 & 0,306 & 0,099 & 32 \\
\hline A/Swine/Germany/R1738/2010 (H1N1av) & 0,209 & 0,112 & 54 & 1,241 & 0,312 & 25 & 0,291 & 0,107 & 37 \\
\hline A/swine/Germany/R1207/2010 (H1N2) & 0,176 & 0,16 & 91 & 0,078 & 0,061 & 78 & 0,087 & 0,06 & 69 \\
\hline A/swine/Germany/R76/2011 (H3N2) & 0,182 & 0,111 & 61 & 0,159 & 0,121 & 76 & 0,768 & 0,147 & 19 \\
\hline
\end{tabular}

${ }^{a}$ Subtype and designation of influenza $A$ virus isolate used to raise the respective serum.

${ }^{b}$ Bold face indicates homologous rec HA1 - serum pair.

AVI (\%) - percentage avidity index.

but had a significant negative influence on homologous HA1-serum reactions as well. Thus, urea treatment grossly decreased sensitivity with no apparent benefit on specificity. In further assays therefore urea washing steps were omitted.

\section{Detection of influenza-specific antibodies in sera of experimentally infected swine}

In a next step the dynamics of antibody development after experimental influenza virus infections of pigs was examined. Experimental infections had been carried out previously using a pandemic $\mathrm{H} 1$ virus (A/sw/Germany/R708/ 2010), an H1pdmN2 reassortant (A/sw/Germany/R2035/ 2011), and an H1N1av isolate (A/sw/Germany/R248/2010). Sera obtained at different time points post infection were available for testing by a commercial NP blocking ELISA (ID.Vet, France), the indirect ELISAs specific for recombinant NP and for the homologous HA1 proteins, respectively. In addition, $\mathrm{HI}$ titers measured against the homologous virus were compared. Results are presented in composite Figure 3. The data show that antibody dynamics in experimentally infected swine can be accurately followed using the indirect ELISA formats. The results compare favorably with the commercial NP blocking ELISA and homologous HI.

\section{Detection of influenza-specific antibodies in porcine field sera}

Finally, reactivity of a collection of 207 porcine field sera was compared by testing sera in HI assays, commercial NP blocking ELISA and indirect ELISAs with recombinant NP and HA1- antigens. These sera represented daily routine diagnostic submissions. As regards the $\mathrm{HI}$ assay, a serum was rated qualitatively positive for influenza A specific antibodies if it showed an HI titre of $\geq 40\left(\geq 3 \log _{2}\right.$, starting dilution 1:10) against at least one of the four antigens (H1N1av, H1N2, H1N1pdm, H3N2) used for testing. Depicting the results of the commercial NP blocking ELISA, the indirect NP ELISA and the sum of the HI assays using a Venn diagram (Figure 4; [24]), an overall high degree of agreement was visually evident. The majority of results was backed by all three or at least two of the assays. However, in the HI assays, a comparatively high number of 26 sera revealed positive results just above the threshold ( 3 or $4 \log _{2}$ ) which were not supported by other assays (Figure 4).

Inter-rater agreement [25] was used to evaluate results of indirect ELISAs using recombinant HA1 compared to HI titres. While excellent and good agreements were seen for the H3 ( $\kappa_{\mathrm{R} 96}=0,8255$ [95\% CI 0,6997-0,9513]) and the pandemic $\mathrm{H} 1$ antigens $\left(\kappa_{\mathrm{R} 26}=0,7772 \quad[0,6917-0,8627]\right.$; $\left.\kappa_{R 2035}=0,661[0,5643-0,7577]\right)$, respectively, only moderate agreement was signaled for the H1av $\left(\kappa_{\mathrm{R} 1738}=\right.$ $0,5722[0,4401-0,7043])$ and H1N2 $\left(\kappa_{\mathrm{R} 1207}=0,5083\right.$ [0,3359$0,6807]$ ) antigens. A graphical analysis revealed that correlation between $\mathrm{HI}$ titres and $\mathrm{S} / \mathrm{P}$ ratios was stronger for higher but less tight for sera with lower HI titres (Figure 5, examplified for recombinant H1av HA1 antigen). A good agreement $\left(\kappa_{\text {HOLDING7 }}=0,625[0,374-0,876]\right)$ across all indirect ELISAs was seen when results were compared based on holding level instead of individual sera (Table 5).

\section{Discussion}

Serodiagnosis of porcine influenza virus infections in Europe and elsewhere is significantly challenged by cocirculation of several subtypes, and use of multivalent vaccines further complicates this situation. While generic antibodies directed against the well conserved influenza virus NP protein can be detected by commercial blocking ELISAs, the differentiation of subtype-specific antibodies requires use of the fastidious HI assay. Subtype-specific ELISA assays suitable for high throughput investigations of porcine sera would aid in promoting more intense studies on porcine influenza seroepidemiology.

Aiming to develop such assays we have successfully expressed recombinant full-length NP and HA1 fragments in bacteria and refolded proteins in vitro. Recombinant proteins were co-translationally mono-bitoinylated which facilitated purification and binding to solid, streptavidincoated supports. Previous work by [26] has shown that 


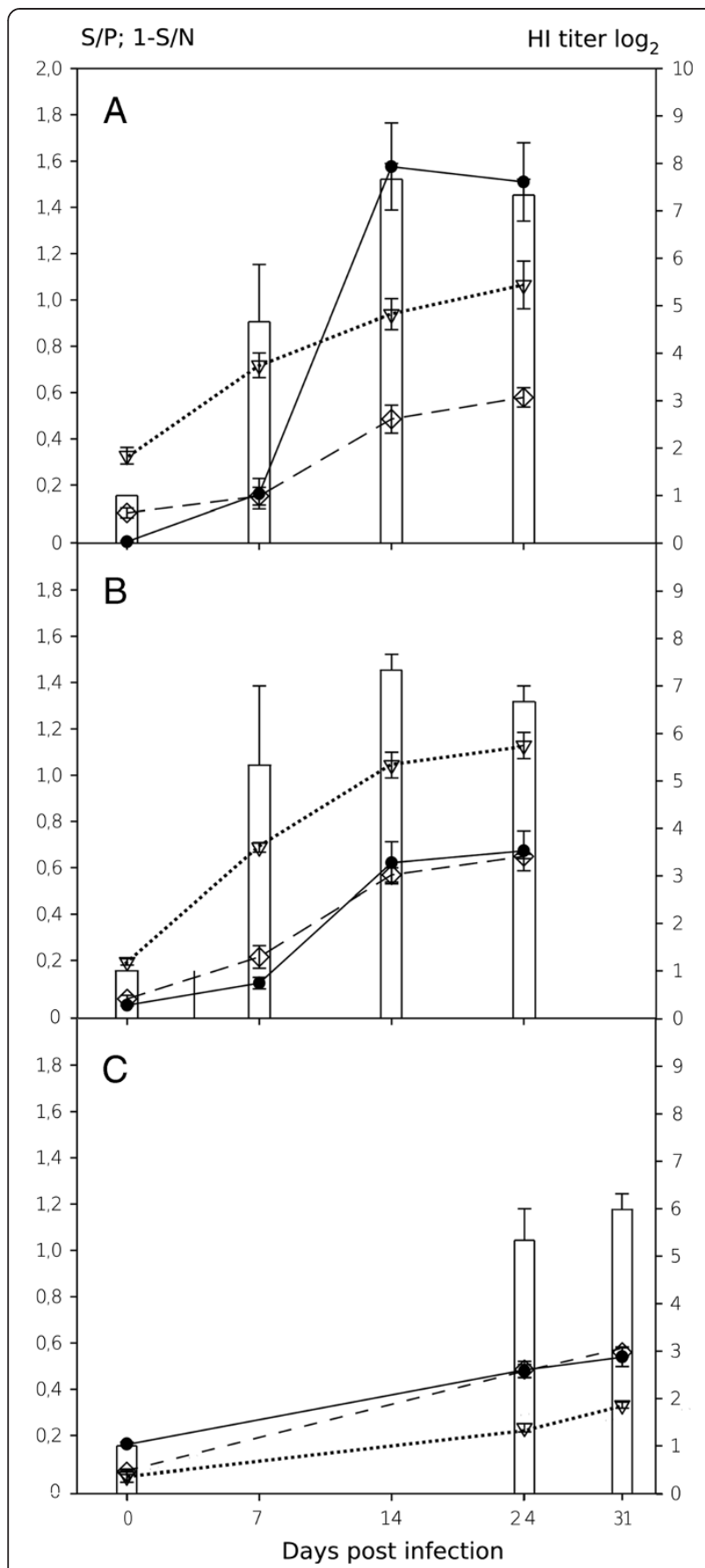

Figure 3 Development of antibodies against viral nucleocapsid and hemagglutinin fragment $\mathrm{HA} 1$ proteins in swine experimentally infected with porcine influenza $A$ viruses. Experimental infection each of three pigs by $\mathbf{A}-\mathrm{A} / \mathrm{sw} / \mathrm{Germany} / \mathrm{R} 708 / 2010$ (H1 pdmN1), B - A/sw/Germany/R2035/2011 (H1 pdmN2), and C - A/sw/Germany/ R248/2010 (H1N1av). Columns - HI titer against homologous virus antigen; open triangle - indirect recombinant NP ELISA; black dot indirect recombinant HA1 ELISA (homologous antigen); open diamond commercial blocking NP ELISA (ID.Vet). Samples of days 7 and 14 were unfortunately not available for experiment " $C$ ". bacterially expressed HA proteins can be refolded to acquire native conformation. Recombinant proteins representing recent isolates of the major subtypes and lineages of porcine influenza virus currently circulating in Germany were recognized by specific porcine or mustelid immune sera in Western blotting and indirect ELISA. Dynamics of generic and subtype-specific antibody development in experimentally infected swine as measured by recombinant indirect ELISAs fully paralleled results obtained by a commercial NP blocking ELISA and homologous HI assays.

However, despite use of the less conserved HA1 section of the HA glycoprotein as diagnostic antigen residual cross reactivity between the different subtypes was noticed in both Western blot (Figure 1) and indirect ELISA (Figure 2) even when using experimental post infection sera which were reasonably discriminatory between subtypes in HI assays (Table 2). Thus, a number of conserved epitopes exists between the different subtypes in the HA1 which is not detected by the functional HI assay. Yet, a considerable number of subtype-specific epitopes must have been represented in the recombinant HA1 proteins as well since, in indirect ELISA, the homologous HA1serum pairs always resulted by far in the highest signal intensity (Figure 2). Human pandemic H1 HA1 represented by isolate $\mathrm{R} 26 / 11$ and that of the porcine-adapted variant H1pdmN2 R2035/11 were indistinguishable by Western blot and indirect ELISA although slight antigenic differences have been reported when using an HI assay [9].

The comparative examination of porcine field sera showed a variable agreement for the different recombinant antigens when individual sera were compared. In particular, ELISAs based on recombinant $\mathrm{H} 1 \mathrm{av}$ and $\mathrm{H} 1 \mathrm{~N} 2$ revealed only moderate agreement when compared to $\mathrm{HI}$. Fewer sera scored positive in the indirect ELISAs compared to HI assays. The majority of sera missed by the indirect ELISA showed low $\mathrm{HI}$ titres (3 or $4 \log _{2}$ ) while reasonable correlation was seen between $\mathrm{S} / \mathrm{P}$ ratios and HI titres for higher-titred sera (Figure 5). The blurring of results obtained with low-titred HI-positive sera may be due to low sensitivity of the indirect ELISAs but could as well have been caused by lack of specificity of the HI assays; problems with reproducibility and standardization of $\mathrm{HI}$ assays are notorious, especially when testing low-titred sera [20]. In addition, the HI assays here were carried out with antigens selected and used in routine diagnosis while the recombinant antigens were produced from more recent circulating viruses and differed slightly in HA1 amino acid sequences (not shown). This may have introduced further discrepancies as observed for individual sera. However, when results were compared on a herd basis a full match between $\mathrm{HI}$ and indirect ELISA results was evident (Table 5). Herds found to be seropositive by $\mathrm{HI}$ for a certain subtype were similarly positive in the respective HA1 indirect ELISA. 


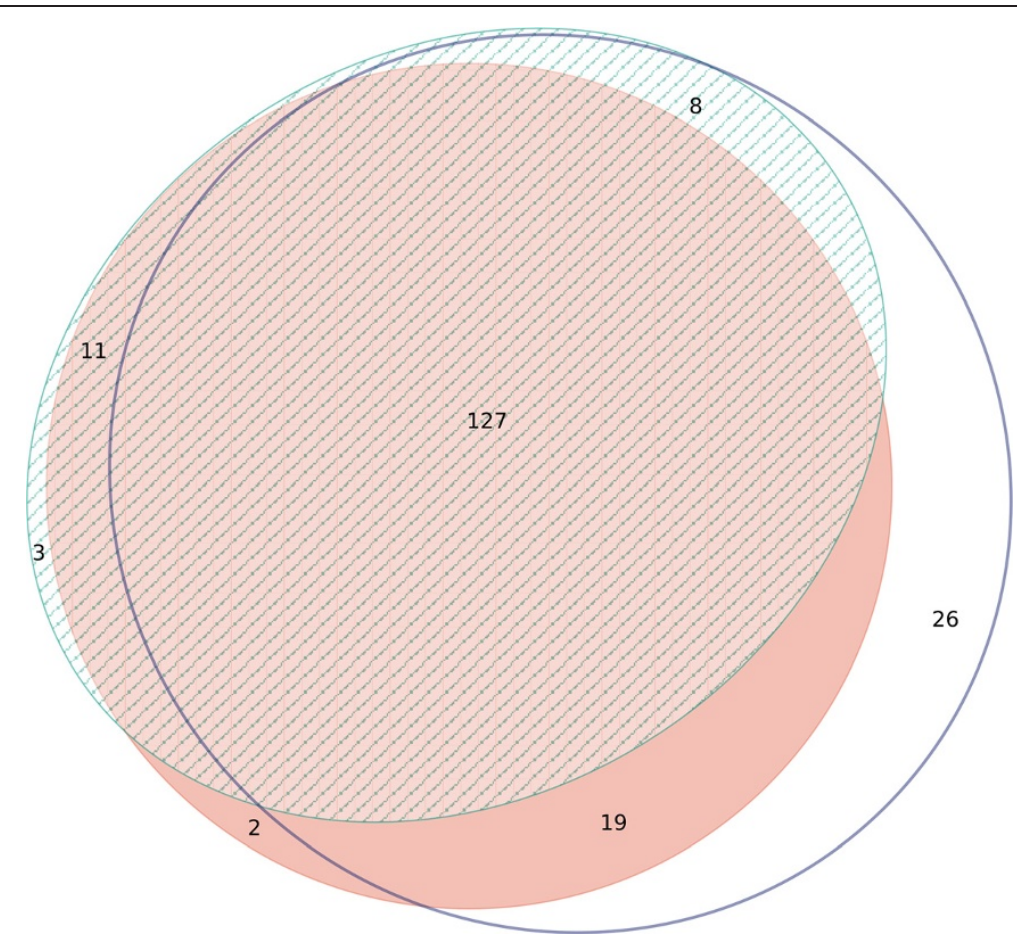

Figure 4 Venn diagram of detection of NP-specific antibodies by commercial blocking ELISA (hatched ellipsis) and indirect ELISA (recombinant NP; red ellipsis) in porcine field sera $(n=207)$ compared with hemagglutination inhibition assays (white ellipsis). Eleven sera produced congruently negative results in all three tests.

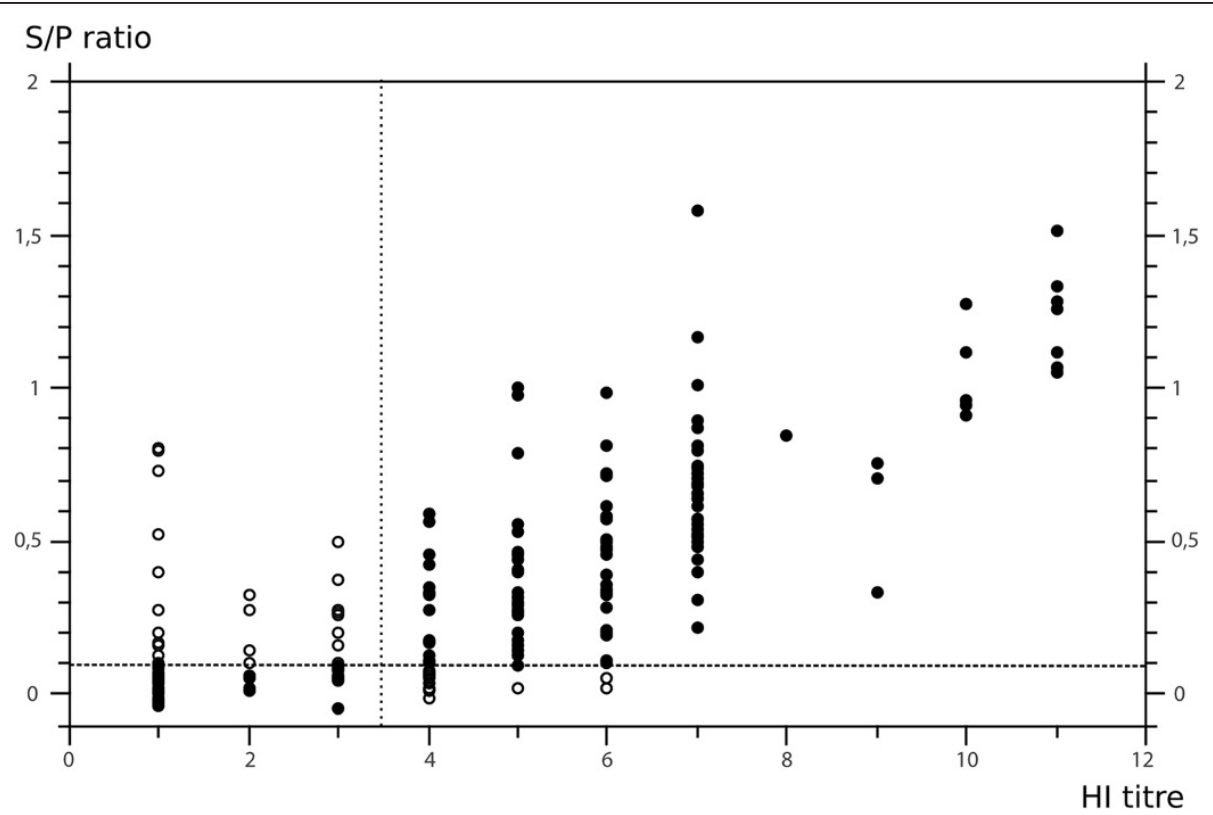

Figure 5 Comparison of $\mathrm{HI}$ titres measured against porcine avian-derived H1N1 isolate A/swine/Germany/R248/2010 and S/P ratios obtained by indirect ELISA using recombinant HA1 antigen of avian-derived H1N1 isolate A/swine/Germany/R1738/2011. Black dots congruent qualitative result; white dots - incongruent qualitative result; HI titre shown as $\log _{2}$ series (starting dilution $1=1: 10$ ); dotted lines represent cut-off values. 
Table 5 Results of recombinant indirect ELISAs (iEIA) and HI or commercial NP-specific ELISA obtained with 207 porcine field sera compared on a holding base

\begin{tabular}{|c|c|c|c|c|c|c|c|c|c|c|c|c|c|}
\hline \multirow[t]{2}{*}{ Holding } & \multirow[t]{2}{*}{ Sample } & \multicolumn{2}{|c|}{ NP } & \multicolumn{2}{|c|}{$\mathrm{H} 1 \mathrm{av}$} & \multicolumn{2}{|c|}{$\mathrm{H} 1 \mathrm{~N} 2$} & \multicolumn{2}{|c|}{ H1pdm } & \multicolumn{2}{|c|}{ H1pdmN2 } & \multicolumn{2}{|c|}{ H3 } \\
\hline & & cEIA IDVet & iEIA & $\mathrm{HI}$ & iEIA & $\mathrm{HI}$ & iEIA & $\mathrm{HI}$ & iEIA & $\mathrm{HI}$ & iEIA & $\mathrm{HI}$ & iEIA \\
\hline 1 & $6^{a}$ & $5^{b}$ & 4 & 5 & 6 & 1 & 0 & 4 & 4 & n.d. & 2 & 0 & 0 \\
\hline 2 & 16 & 15 & 12 & 14 & 16 & 3 & 0 & 13 & 16 & n.d. & 16 & 6 & 6 \\
\hline 3 & 9 & 7 & 7 & 7 & 7 & 9 & 2 & 3 & 6 & n.d. & 4 & 5 & 3 \\
\hline 4 & 7 & 7 & 7 & 7 & 7 & 7 & 7 & 7 & 7 & n.d. & 7 & 7 & 7 \\
\hline 5 & 5 & 5 & 5 & 5 & 5 & 5 & 5 & 5 & 5 & n.d. & 5 & 5 & 5 \\
\hline 6 & 30 & 30 & 28 & 30 & 29 & 1 & 0 & 28 & 26 & n.d. & 20 & 0 & 3 \\
\hline 7 & 30 & 24 & 26 & 22 & 22 & 3 & 1 & 17 & 15 & n.d. & 14 & 0 & 0 \\
\hline 8 & 30 & 13 & 18 & 8 & 14 & 0 & 0 & 1 & 3 & n.d. & 3 & 0 & 0 \\
\hline 9 & 30 & 18 & 28 & 28 & 26 & 0 & 1 & 26 & 21 & n.d. & 15 & 0 & 0 \\
\hline 10 & 23 & 3 & 12 & 9 & 7 & 3 & 0 & 0 & 0 & n.d. & 0 & 1 & 0 \\
\hline 11 & 13 & 11 & 12 & 13 & 13 & 3 & 1 & 12 & 11 & n.d. & 6 & 0 & 0 \\
\hline 12 & 10 & 10 & 10 & 10 & 6 & 1 & 1 & 5 & 3 & n.d. & 3 & 0 & 0 \\
\hline
\end{tabular}

${ }^{a}$ Number of sample obtained in holding.

${ }^{b}$ Number of sample rated positive in the respective assay. n.d. Not determined.

Moreover, herds negative by $\mathrm{HI}$ for a certain subtype tested negative by the corresponding indirect ELISA. This indicates that on herd base the HI assay may be replaced, without loss of diagnostic quality, by the indirect ELISAs.

\section{Conclusions}

Due to the ongoing antigenic diversification of porcine influenza viruses worldwide and new reassortant lineages springing up here and there serological diagnosis of porcine influenza becomes ever more demanding. Subtype and lineage-specific assays suitable for high throughput analysis will be required to cope with such diagnostic challenges. The recombinant mono-biotinylated HA1 proteins presented here as diagnostic antigens in indirect ELISAs provided an interesting alternative in this respect to HI assays. Further refinements of this strategy, e.g., by using lineage-specific monoclonal antibodies for competition or an immune-complex binding assay format, should be further investigated to replace $\mathrm{HI}$ assays.

\section{Material and methods}

Virus and cell culture

Influenza A viruses were propagated in serum-free MDCK cell cultures in the presence of TPCK-trypsin as detailed elsewhere [11]. Isolates were obtained from the virus repository maintained at the Friedrich-Loeffler-Institut. Molecular characteristics of recent porcine field isolates have previously been reported [9]. A list of viruses used in this study for production of recombinant proteins is provided in Table 1.

Bacterial expression, in vivo biotinylation and purification of influenza virus HA1 and NP proteins

The HA1 fragments of the viral hemagglutinin openreading frames (ORF) were cloned into the $\mathrm{pET} 19 \mathrm{~b}$ vector by a target-primed technique using Phusion polymerase amplification and $D p n$ I digested amplificates [27]. Sequences of primers are available on request. Expressed sequences stretched from the first amino acid of the mature protein to the arginin residue immediately proximal to the first glycin residue of the HA2 fusion peptide. Downstream of this arginin residue an Avi-Tag consensus sequence [28] was inserted. The central lysin residue of the 15 amino acid Avi-Tag sequence provides an acceptor site for covalent linkage of D-biotin which is specifically catalyzed by the bacterial biotin transferase BirA [29].

HA1-pET19b expression constructs were co-transformed into Rosettagami $E$. coli with plasmid pBIRAcm (Avidity, Aurora, CO, U.S.A.) for overexpression of BirA. Dually transformed cells were selected using ampicillin and chloramphenicol (CM). Since CM is also required to maintain the genotype of Rosettagami $E$. coli cells, presence of both plasmids in selected colonies had to be confirmed by plasmid/insert-specific PCRs (primer sequences available on request). TYH medium supplemented with D-biotin at a concentration of $50 \mu \mathrm{g} / \mathrm{ml}$ was used for expression of co-translationally mono-biotinylatied Avi-tagged 
recombinant protein. The full length ORF of the nucleocapsid gene of the porcine influenza virus isolate R1738/10 was cloned and expressed similarly. However, the Avi-Tag was placed at the N-terminus of the protein. Lysates of Rosettagami cells transformed with plasmid pBIRAcm and an empty pET19b vector were used as a negative expression control.

Monobiotinylated bacterially expressed recombinant proteins were purified from inclusion bodies (IBs) by centrifugation and washing steps as previously described [26]. Proteins sequestered in purified IBs were then subjected to solubilization in $6 \mathrm{M}$ guanidin- $\mathrm{HCl}$ and refolding using a panel of up to 30 different primary and up to nine secondary buffer conditions in a stepwise solubilization strategy using the ProteoStat kit (Enzo, Lörrach, Germany). Protein solubilization and reactivity were screened with conformation-dependent monoclonal antibodies and specific polyclonal sera in ELISA to sort out optimal refolding conditions for each of the recombinantly expressed proteins. Here, refolding conditions were used which had been validated using avian influenza virus H5 HA1 protein and two conformationdependent monoclonal antibodies, 3H12 and 5 F3 (see [30], for properties of monoclonal antibodies). Final concentrations of recombinant proteins in appropriate refolding buffers were measured using a Coomassie protein assay kit (ThermoScientific, Rockford, IL, U.S.A.).

\section{Production of subtype-specific antisera}

Pigs or ferrets were experimentally infected by the oronasal route with $10^{6} \mathrm{TCID}_{50}$ of MDCK cell culture-grown influenza viruses in $1 \mathrm{ml}$ cell culture medium using a nebulizer device (Wolfe Tory Medical, Salt Lake City, Utah) as previously described [31]. All experiments had received legal approval by an ethics commission (LALLF M-V/TSD/7221.3-2.5-004/10). Prior to infection animals were tested seronegative for influenza NP-specific antibodies in a commercial blocking ELISA (ID.Vet). Virus isolates used for infection are listed in Table 2. Blood samples used in further serological studies were obtained on day 21 post inoculation (p.i.). Reactivity of post infection sera in hemagglutination inhibition assays is detailed in Table 2.

\section{Origin of field sera}

Porcine field sera from 12 swine holdings in Germany were submitted for routine diagnostic procedures. The history of these holdings for vaccination against influenza and/or clinical episodes of influenza virus infection was not documented.

\section{Indirect ELISA}

Bacterially expressed proteins in refolding buffer were adjusted to a concentration of $5 \mu \mathrm{g} / \mathrm{ml}$ using TRIS- buffered saline (TBS). A total of $100 \mu \mathrm{l}$ per well was used for binding to streptavidin-coated plates for 2 hours at room temperature or overnight at $4^{\circ} \mathrm{C}$. Each plate included different recombinant antigens for each row with following strains: A:SIV/R1738/10 (H1N1),B: SIV/ R1207/ 11 (H1N2),C: -SIV/ R26/11 (H1N1pdm)D: SIV R2035/11 H1pdmN2, E: SIV/- R1931 /11 (H1N2), F: R76/11 (H3N2), G: R96/11 (H3N2), H: R1738/10 nucleocapsid protein. After washing wells were blocked using 5\% nonfat milkTBS containing 0.05\% Tween 20 (TBST) (see Postel et al., 2011) for 2 hours at room temperature and then washed four times with TBST. Individual sera $(100 \mu \mathrm{l}$ per well, prediluted 1:200 in sample dilution buffer [ID.Vet, Montpellier, France]) were pipetted into columns $(1 \mathrm{~A}-1 \mathrm{H})$ of the microtitre plate. This procedure assured that the reactivity of each serum against all antigens was measured in the same plate. Sera were incubated at room temperature for 1 hour. Wells were washed again four times with TBST before $100 \mu \mathrm{l}$ of appropriately diluted goat-anti-swine IgG peroxidase conjugate (Dianova) was added for one hour at room temperature. Antibody was removed and after a final washing cycle with TBST, $50 \mu \mathrm{l}$ of chromogenic TMB substrate was added. $\mathrm{OD}_{450}$ values were measured after 10 minutes of incubation and addition of $50 \mu \mathrm{l}$ of $1 \mathrm{~N} \mathrm{H}_{2} \mathrm{SO}_{4}$ to each well. Results were calculated and expressed in S/P units:

$$
\frac{\text { OD Test-ODBackground }}{\text { ODPositive control-ODBackground }} \times 100=\mathrm{S} / \mathrm{P}
$$

\section{Avidity measurement of sera by indirect ELISA}

The indirect ELISA was performed as described above. However, after incubation of sera in the wells a washing step using urea in TBST was carried out. Different urea concentrations $(0.5$ and $2 \mathrm{M})$ and incubation times were evaluated, Final assays were carried out with $6 \mathrm{M}$ urea for ten minutes at room temperature. Consecutive washing steps and conjugate incubation were carried out with TBST without urea. The sera were tested in parallel with and without the urea-buffer washing step and an avidity index (AVI) was calculated:

$$
\frac{\text { OD sample without urea }}{\text { OD sample with urea }} \times 100=\mathrm{AVI}
$$

\section{Generic nucleocapsid protein blocking ELISA (NP-bEIA)}

For detection of group specific antibodies a commercial NP-bEIA was purchased (ID.Vet, Montpellier, France) and used according to recommendations of the manufacturer. Accordingly, samples were considered positive if the $\mathrm{S} / \mathrm{N}$ (sample $\mathrm{OD}_{450} /$ negative-control $\mathrm{OD}_{450} \times 100$ ) ratio was less than $45 \%$, negative if the $\mathrm{S} / \mathrm{N}$ ratio was more than $50 \%$, and doubtful if the $\mathrm{S} / \mathrm{N}$ ratio was between $45 \%$ and $50 \%$. 


\section{Hemagglutination inhibition assay $(\mathrm{HI})$}

$\mathrm{HI}$ assays were performed according to O.I.E. recommendations essentially as described by [31]. Four hemagglutinating units of cell culture-grown influenza viruses were used throughout. All porcine and ferret sera were heat-inactivated for 30 minutes at $56^{\circ} \mathrm{C}$ and treated with receptor-destroying enzyme (neuraminidase from Bacillus subtilis).

\section{Abbreviations}

AVI: Avidity index; IAV: Influenza A virus; H1av: Avian-derived porcine subtype $\mathrm{H1}$; H1 pdm: Human pandemic 2009-derived porcine subtype $\mathrm{H} 1$; HA: Hemagglutinin protein; HA1: Hemagglutinin fragment-1 protein; HI: Hemagglutination inhibition assay; NA: Neuraminidase protein; NP: Nucleocapsid protein; RT-qPCR: Real time reverse transcription polymerase chain reaction; TBS: TRIS-bufferdd saline; TBST: TRIS-buffered saline with Tween 20 supplement; TMB: 3,3',5,5'-Tetramethylbenzidine.

\section{Competing interests}

The authors declare that they have no competing interests.

\section{Authors' contributions}

ZN, CG and TCH conceived the study and drafted the manuscript. ZN carried out the molecular and serological work, and analysed samples. SK participated in the molecular and serological work. EL carried out animal experiments and provided samples. MB conceived the study, provided funds and edited the manuscript. All authors read and approved the final manuscript.

\section{Acknowledgements}

Z.N. is recipient of a fellowship of the China Scholarship Council (CSC). The study has been supported through FSI projects 2.1 .1 and 3.2 by the German Federal Ministry for Food, Agriculture and Consumer Protection, and by a grant from vaxxinova, Münster, Germany. The FLI is an associated member of the EU-FP7 project ESNIP-3.

\section{Author details}

'Institute of Diagnostic Virology, Friedrich-Loeffler-Institut, Suedufer 10, Greifswald 17493, Germany. ${ }^{2}$ Department of Experimental Animal Facilities and Biorisk Management, Friedrich-Loeffler-Institut, Suedufer 10, Greifswald 17493, Germany. ${ }^{3}$ Institute of Immunology, Friedrich-Loeffler-Institut, Suedufer 10, Greifswald 17493, Germany.

Received: 2 April 2013 Accepted: 22 July 2013

Published: 30 July 2013

\section{References}

1. Torremorell M, Allerson M, Corzo C, Diaz A, Gramer M: Transmission of influenza a virus in pigs. Transbound Emerg Dis 2012, 59(s1):63-84.

2. Opriessnig T, Giménez-Lirola LG, Halbur PG: Polymicrobial respiratory disease in pigs. Anim Health Res Rev 2011, 12:133-148.

3. Kyriakis CS, Rose N, Foni E, Maldonado J, Loeffen WL, Madec F, Simon G, Van Reeth K: Influenza A virus infection dynamics in swine farms in Belgium, France, Italy and Spain, 2006-2008. Vet Microbiol 2013, 162:543-550.

4. Chen Q, Madson D, Miller CL, Harris DL: Vaccine development for protecting swine against influenza virus. Anim Health Res Rev 2012, 13:181-95.

5. Trebbien R, Larsen LE, Viuff BM: Distribution of sialic acid receptors and influenza A virus of avian and swine origin in experimentally infected pigs. Virol J 2011, 8:434.

6. Ma W, Lager KM, Vincent AL, Janke BH, Gramer MR, Richt JA: The role of swine in the generation of novel influenza viruses. Zoonoses Public Health 2009, 56:326-337.

7. Hass J, Matuszewski S, Cieslik D, Haase M: The role of swine as "mixing vessel" for interspecies transmission of the influenza A subtype H1N1: a simultaneous Bayesian inference of phylogeny and ancestral hosts. Infect Genet Evol 2011, 11:437-441.

8. Brown IH: History and epidemiology of swine influenza in Europe. Curr Top Microbiol Immunol 2012. [Epub ahead of print] PubMed PMID: 22234411.
9. Starick E, Lange E, Grund C, Grosse Beilage E, Döhring S, Maas A, Noé T, Beer M, Harder TC: Reassortants of pandemic influenza A virus H1N1/2009 and endemic porcine $\mathrm{HxN} 2$ viruses emerge in swine populations in Germany. J Gen Virol 2012, 93:1658-1663.

10. Harder TC, Reuß A, Buda S: Zoonotic influenza [ger. Influenza als Zoonose]. In Epidemiological report on influenza in Germany. 12th edition. Berlin, Germany: Robert-Koch-Institut; 2011:75-81. Accessed: http://edoc.rki. de/series/Trash/PDF/abschlussbericht_2012.pdf

11. Starick E, Lange E, Fereidouni S, Bunzenthal C, Höveler R, Kuczka A, Grosse Beilage E, Hamann HP, Klingelhöfer I, Steinhauer D, Vahlenkamp T, Beer M, Harder T: Reassorted pandemic (H1N1) 2009 influenza A virus discovered from pigs in Germany. J Gen Virol 2011, 92:1184-1188.

12. Brookes SM, Núñez A, Choudhury B, Matrosovich M, Essen SC, Clifford D, Slomka MJ, Kuntz-Simon G, Garcon F, Nash B, Hanna A, Heegaard PM, Quéguiner S, Chiapponi C, Bublot M, Garcia JM, Gardner R, Foni E, Loeffen W, Larsen L, Van Reeth K, Banks J, Irvine RM, Brown IH: Replication, pathogenesis and transmission of pandemic (H1N1) 2009 virus in non-immune pigs. PLoS One 2010, 5:e9068

13. Starick E, Fereidouni SR, Lange E, Grund C, Vahlenkamp T, Beer M, Harder TC. Analysis of influenza $\mathrm{A}$ viruses of subtype $\mathrm{H} 1$ from wild birds, turkeys and pigs in Germany reveals interspecies transmission events. Influenza Other Respi Viruses 2011, 5:276-284.

14. Detmer S, Gramer M, Goyal S, Torremorell M, Torrison J: Diagnostics and surveillance for swine influenza. Curr Top Microbiol Immunol 2012. [Epub ahead of print] PubMed PMID: 22566130.

15. Sreta $D$, Jittimanee $S$, Charoenvisal N, Amonsin A, Kitikoon $P$, Thanawongnuwech R: Retrospective swine influenza serological surveillance in the four highest pig density provinces of Thailand before the introduction of the 2009 pandemic Influenza A virus subtype H1N1 using various antibody detection assays. J Vet Diagn Invest 2013, 25:45-53.

16. Loeffen WL, Nodelijk G, Heinen PP, van Leengoed LA, Hunneman WA, Verheijden $\mathrm{JH}$ : Estimating the incidence of influenza-virus infections in Dutch weaned piglets using blood samples from a cross-sectional study. Vet Microbiol 2003, 91:295-308.

17. Loving CL, Vincent AL, Pena L, Perez DR: Heightened adaptive immune responses following vaccination with a temperature-sensitive, live-attenuated influenza virus compared to adjuvanted, whole-inactivated virus in pigs. Vaccine 2012, 30:5830-5838.

18. Ciacci-Zanella JR, Vincent AL, Prickett JR, Zimmerman SM, Zimmerman JJ: Detection of anti-influenza A nucleoprotein antibodies in pigs using a commercial influenza epitope-blocking enzyme-linked immunosorbent assay developed for avian species. J Vet Diagn Invest 2010, 22:3-9.

19. Katz JM, Hancock K, Xu X: Serologic assays for influenza surveillance, diagnosis and vaccine evaluation. Expert Rev Anti Infect Ther 2011, 9:669-683.

20. Wood JM, Montomoli E, Newman RW, Daas A, Buchheit KH, Terao E: Collaborative study on influenza vaccine clinical trial serology - part 2: reproducibility study. Pharmeur Bio Sci Notes 2011, 1:36-54.

21. Kim WI, Wu WH, Janke B, Yoon KJ: Characterization of the humoral immune response of experimentally infected and vaccinated pigs to swine influenza viral proteins. Arch Virol 2006, 151:23-36.

22. Lang C, Sipos W, Dürrwald R, Herwig V, Selbitz HJ, Sommerfeld-Stur I, Schmoll F: Hämagglutinationsinhibitiontest und kommerzielle ELISA-Kits zum Nachweis von Antikörpern gegen Schweineinfluenzavirus TypA-H1N1- und -H3N2 differieren in ihrer diagnostischen Aussagekraft [ger. Hemagglutination inhibition assay and commercial ELISA for detection of antibodies against porcine influenza virus subtype $\mathrm{H} 1 \mathrm{~N} 1$ and $\mathrm{H} 3 \mathrm{~N} 2$ differ in their diagnostic significance.]. Wien Tierärztl Mschr 2007, 94:296-303.

23. Barbé $F$, Labarque $G$, Pensaert $M$, Van Reeth $K$ : Performance of a commercial swine influenza virus $\mathrm{H} 1 \mathrm{~N} 1$ and $\mathrm{H} 3 \mathrm{~N} 2$ antibody enzymelinked immunosorbent assay in pigs experimentally infected with European influenza viruses. J Vet Diagn Invest 2009, 21:88-96.

24. Rodgers P, Flower J, Stapleton G, Howse J: Drawing area-proportional venn-3 diagrams with convex polygons. Portland, OR, USA: Diagrammatic Representation and Inference, 6th International Conference, Diagrams 2010; 2010. Proceedings; 01/2010.

25. Fleiss JL, Cohen J, Everitt BS: Large sample standard errors of kappa and weighted kappa. Psychological Bull 1969, 72:323-327.

26. Khurana S, Larkin C, Verma S, Joshi MB, Fontana J, Steven AC, King LR, Manischewitz J, McCormick W, Gupta RK, Golding H: Recombinant HA1 produced in E. coli forms functional oligomers and generates strain-specific SRID potency antibodies for pandemic influenza vaccines. Vaccine 2011, 29:5657-5665. 
27. Stech J, Stech O, Herwig A, Altmeppen H, Hundt J, Gohrbandt S, Kreibich A, Weber S, Klenk HD, Mettenleiter TC: Rapid and reliable universal cloning of influenza A virus genes by target-primed plasmid amplification. Nucleic Acids Res 2008, 36: 139

28. Rigaut G, Shevchenko A, Rutz B, Wilm M, Mann M, Seraphin B: A generic protein purification method for protein complex characterization and proteome exploration. Nat Biotechnol 1999, 17:1030-1032.

29. Beckett D, Kovaleva E, Schatz PJ: A minimal peptide substrate in biotin holoenzyme synthetase-catalyzed biotinylation. Protein Sci 1999, 8:921-929.

30. Postel A, Ziller M, Rudolf $M$, Letzel T, Ehricht R, Pourquier P, Dauber M, Grund C, Beer M, Harder TC: Broad spectrum reactivity versus subtype specificity-trade-offs in serodiagnosis of influenza $A$ virus infections by competitive ELISA. J Virol Methods 2011, 173:49-59.

31. Lange E, Kalthoff D, Blohm U, Teifke JP, Breithaupt A, Maresch C, Starick E, Fereidouni S, Hoffmann B, Mettenleiter TC, Beer M, Vahlenkamp TW: Pathogenesis and transmission of the novel swine-origin influenza virus A/H1N1 after experimental infection of pigs. J Gen Virol 2009, 90:2119-2123.

doi:10.1186/1743-422X-10-246

Cite this article as: Zhao et al:: Distinction of subtype-specific antibodies against European porcine influenza viruses by indirect ELISA based on recombinant hemagglutinin protein fragment-1. Virology Journal 2013 10:246

\section{Submit your next manuscript to BioMed Central and take full advantage of:}

- Convenient online submission

- Thorough peer review

- No space constraints or color figure charges

- Immediate publication on acceptance

- Inclusion in PubMed, CAS, Scopus and Google Scholar

- Research which is freely available for redistribution 